\title{
REM Sleep Behaviour Disorder in a Child with Tourette's Syndrome
}

\author{
Nikola N. Trajanovic, Inna Voloh, Colin M. Shapiro, Paul Sandor
}

\begin{abstract}
Purpose: To describe an association of Tourette's syndrome with rapid eye movement sleep behaviour disorder (RBD) in a prepubescent boy. Methods: A four year longitudinal single-case study. Results: The co-existence of Tourette's syndrome and RBD was confirmed after polysomnographic studies using the standard criteria. The authors propose possible overlap in the pathophysiological mechanisms underlying the two disorders.
\end{abstract}

RÉSUMÉ: Trouble de comportement du sommeil REM chez un enfant atteint du syndrome de Gilles de la Tourette: cas clinique. Objectif: Décrire l'association d'un syndrome de Gilles de la Tourette et d'un trouble de comportement du sommeil REM (TCSR) chez un jeune garçon. Méthode : Il s'agit d'une étude longitudinale d'un seul cas, sur une période de 4 ans. Résultats : La coexistence du syndrome de Gilles de la Tourette et de TCSR a été confirmée au moyen d'études polysomnographiques utilisant les critères standards. Les auteurs proposent qu'il existe un chevauchement possible dans la physiopathologie sous-jacente à ces deux maladies

Can. J. Neurol. Sci. 2004; 31: 572-575

A number of studies have documented a variety of sleep disorders associated with Tourette's syndrome, ${ }^{1-6}$ including higher incidence of parasomnias, and periodic limb movement disorder ${ }^{7,8}$ persisting both in rapid eye movement (REM) and non-REM sleep. ${ }^{8-11}$ Some researchers also reported increased general movements in REM sleep. ${ }^{12}$ Previously, there was no report of REM sleep behaviour disorder (RBD) in this population, and only two single case history reports existed ${ }^{13,14}$ describing RBD in children, until our initial description of a series of pre-pubescent patients with Tourette's syndrome ${ }^{15}$ and a report of RBD in otherwise asymptomatic children. ${ }^{16}$

In our previous work ${ }^{15}$ we presented a series of 16 patients with Tourette's syndrome, age 6-65 (14 males) who were referred to our clinic for a variety of sleep-related complaints, but predominantly for suspected nocturnal movement disorder (periodic leg movements in sleep (PLMS), restless legs syndrome (RLS), nocturnal tics) and parasomnic behaviour. In 12 patients we observed raised muscle activity in REM sleep, either in the form of (periodic) leg movements or attenuated tics, or (majority) as augmented phasic electromyograph (EMG) without associated movements. Three of these patients had confirmed, and two strongly possible RBD. Following the initial series of patients, further patients with the various sleep pathology were seen, including the case presented in this article.

\section{Case history}

We present a case of a seven-year-old boy with Tourette's syndrome and RBD. The patient was seen over the four-year period starting in 1998 when he was three years old. Polysomnographic (PSG) sleep assessments were done in late 1999 and mid-2002.
YMT is of Chinese extraction. He first exhibited Tourette's syndrome symptoms at the age of two, which consisted of labial tics, sticking the tongue out, jerking shoulders, flexing arms and twirling hair. Some of the simple vocal tics were also present (repeating simple words, such as 'me-me-me', similar to singing). The severity of his motor tics were rated as '4' on a 5-point Tourette's Syndrome Global Scale ${ }^{17}$ ('almost never gone without tics, infrequent tic-free intervals'), and of vocal tics as ' 2 ' on a 5 -point scale ('frequently gone without'). There was no evidence that the onset of symptoms was related to streptococcal infection. The onset of motor and vocal tics was followed by behavioural changes (excessive clinging and attachment to adults, unusual fears, panicky reactions) and sleep disturbances (difficulties falling asleep and frequent awakenings). Symptoms of obsessive compulsive disorder were not present, but he did exhibit symptoms of attention deficit hyperactivity disorder - these were mostly in the sphere of impulsivity, and less in the sphere of inattention.

The patient's parents were aware of his restless sleep and reported that he was 'turning and kicking in his sleep, occasionally making moaning noises or babbling'.

His perinatal history and early development were largely unremarkable. Family history was positive for transient tics (maternal uncle). Physical examination was also unremarkable. YMT was medication free during the period described in this report, partly as a result of his parents'reluctance to expose him to medication.

From the Sleep and Alertness Clinic, (NNT, IV, CMS); Tourette Syndrome Neurodevelopmental Clinic, (PS); University Health Network, Toronto, ON Canada. ReCEIVED FEBRUARY 18, 2004. ACCEPTEDIN FINALFORM JunE 22, 2004. Reprint requests to: N. Trajanovic, Sleep and Alertness Clinic, Med West Medical Centre, Box 22, 221-750 Dundas St., Toronto, ON M6J 3S3 Canada. 
YMT underwent an electroencephalographic (EEG) assessment in 1998. The five-hour monitoring did not show any electroencephalographical seizures. The EEG revealed only nonspecific bilateral frontal and temporal theta rhythms during wakefulness, unrelated to body shaking and shoulder jerking. The CT scan was also done, showing no abnormalities.

At the age of five, after a bout of fever, his tics worsened. At the same time, his behavioural problems and sleep difficulties mitigated after the initiation of the play therapy with a child psychologist, who concluded that these behavioural changes were a result of maladaptive reaction to his tic disorder.

In late 1999, YMT underwent his first two overnight sleep studies. The studies showed a relatively normal sleep onset latency, normal sleep efficiency $(>95 \%)$ and duration ( $>8 \mathrm{~h}$ total sleep time), delayed and reduced REM sleep and relatively normal slow-wave sleep (SWS) for gender and age. The number of spontaneous arousals was slightly increased $(\approx 8 /$ hour), and the number of respiratory events was on high end of normal range for his age group ( 0.5 and 1.4 /hour on respective nights), with normal oxygen saturation in sleep. Periodic leg movements index was 8 . Alpha EEG activity was slightly raised (rated as ' $3 / 5$ '). His tics were attenuated during sleep and present only as electromyographic phenomena. YMT's EMG trace also showed loss of muscle atonia during REM sleep and, in one of the REM periods towards the end of his second night, he had a parasomnic episode where he flailed his arms and right leg in a manner of fending off someone, mumbling fearfully (Figure). Corresponding PSG record showed findings typical of RBD and not associated with sleep-disordered breathing or indicating seizure activity. Also, there was electrophysiological evidence of persistent loss of muscle atonia in REM sleep seen in both the chin EMG and limb EMG channels unrelated to this parasomnic episode. At the same time, a number of abrupt arousals from SWS were seen on both nights, with no behavioural substrate. The EEG trace (standard PSG montage) did not reveal any abnormalities that would suggest seizure disorder. During the post-sleep briefing he said that he was not sure (not dismissing) if he had any dreams on these two occasions.

Subsequently, YMT continued to exhibit motoric tics, which were most prominent just prior to settling to sleep. There was a typical pattern of waxing and waning of symptoms, with relapses mostly associated with episodes of increased stress or febrile illness. Extensive psychometric tests done in 2001 (age six years four months) revealed a wide discrepancy between verbal scores in the low-average range (11th percentile) and performance score in the average range (66th percentile). Academic performance also ranged widely, e.g. basic skills in reading and math were at 93rd and 77th percentile respectively, while receptive language skills were at 21 st percentile, and solving mathematical problems presented in verbal at 8 th percentile. (Wechsler Individual Achievement Test 2 nd ed.) The above results cast doubt on the diagnosis of attention deficit hyperactivity disorder as his learning difficulties were attributed to the receptive language learning disability and his perfectionistic attitude. Other behavioural problems were viewed as part of the Tourette's syndrome symptomatology.

Follow-up sleep assessment was done in summer of 2002. This time extensive EEG monitoring was supplemented with multi-angle videosurveillance. The latter revealed exacerbated motoric tics during the presleep period, intermittent tic activity predominantly in non-SWS nonREM sleep, a brief confusional arousal and no parasomnic activity in REM sleep. The EMG trace again showed loss of atonia during REM. The EEG trace did not show evidence of seizure activity in sleep. In his post-sleep questionnaire he reported having only pleasant dreams.

YMT's parents have not systematically observed their son's nocturnal behaviour. They are aware that, on 'rare occasions', he does move in his sleep, flail his hands and vocalise. His sleep is still restless,

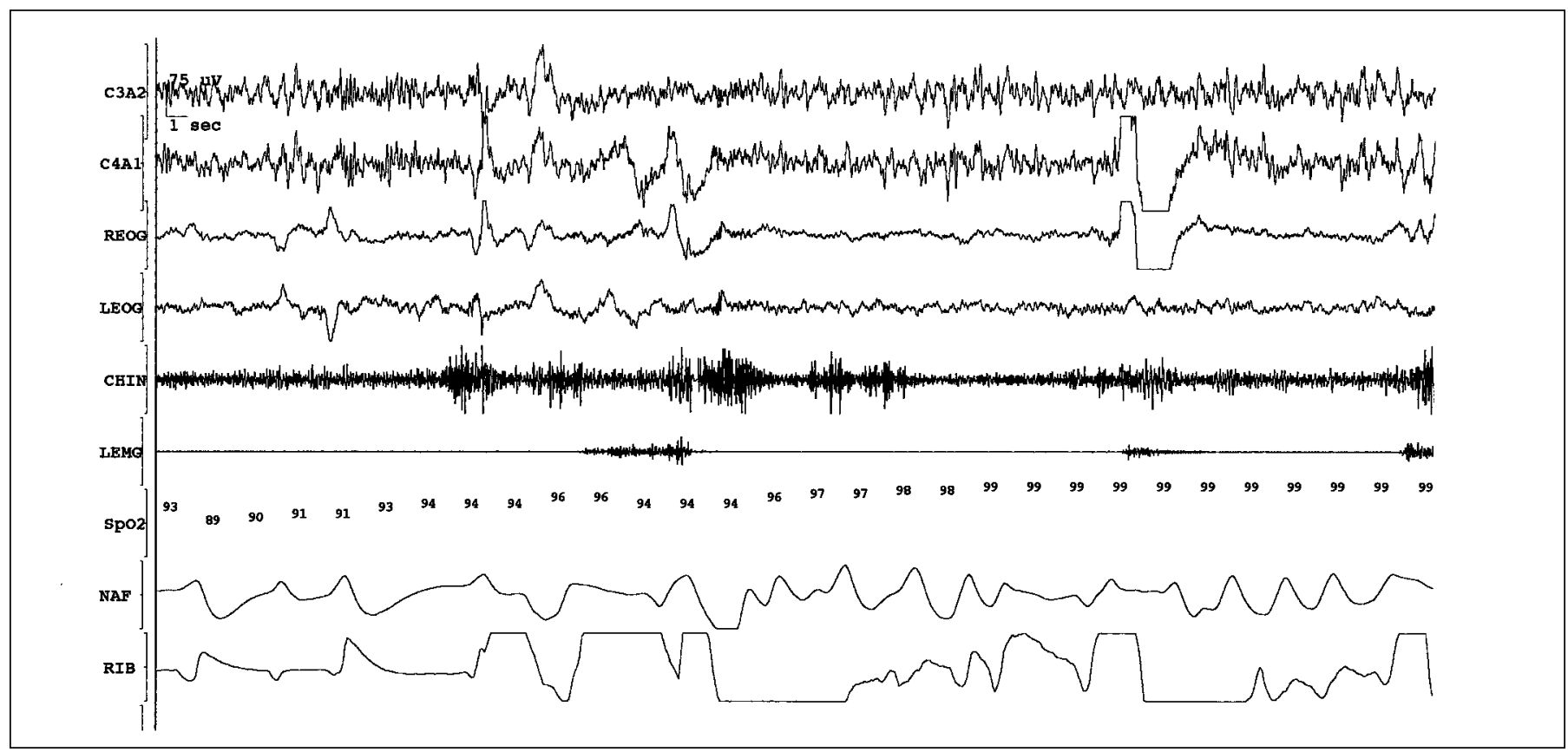

Figure: YMT (age 5) selected REM sleep epoch (note - O, drop due to the movement artefact). Channel labels: C3A2, C4A1 (EEG), REOG, LEOG (eyes), chin (EMG, m. submentalis), LEMG (EMG, m. tibialis anterior bilaterally), $\mathrm{SpO}_{2}$ (arterial oxygen saturation), NAF (nasal flow thermistor), RIB (thoracic respiratory effort) 
but less so when compared to the period of the previous sleep assessment.

The patient remains in care of the Tourette's syndrome specialist. Behavioural interventions and appropriate psychotherapy remain the focus of the management. Because of the nonviolent and sporadic nature of parasomnic episodes, no pharmacological treatment was pursued.

\section{Discussion}

This case illustrates the association between Tourette's syndrome and abnormal sleep physiology. In this case we observed typical features of Tourette's syndrome, and also sleep abnormalities, which included mild sleep fragmentation, variable reduction in sleep efficiency, fluctuating amount of SWS and mildly reduced REM sleep. We have also found presence of mild PLMS and perseverance of tics in sleep, this in reduced and attenuated form. From the perspective of the sleep clinician, the most interesting point is the subtle presence of the genuine REM sleep parasomnia in the form of the REM sleep behaviour disorder. Normally, skeletal muscle atonia during REM sleep is achieved through an active process of motor inhibition by centres located in pontine region, by excitation of the nucleus reticularis magno-celluralis in the medulla, which then hyperpolarises spinal motor pathways. When this circuitry is damaged, as is the case of neurodegenerative diseases and neoplastic processes, or modulated through use of certain psychotropic substances (most notably tricyclic antidepressants and monoamine oxidase inhibitors), generalised REM atonia is replaced by excessive augmentation of muscle tone and, to a high degree repetitive and stereotypical, limb or body movements usually associated with disturbing dream mentation, but with minimal autonomic activation. ${ }^{18}$ In this case the clinical expression and severity of the RBD coincided with the clinical expression of the PLMS. This implies the notion that the pattern of waxing and waning of tics may also apply to the RBD (and possibly PLMS) in Tourette's syndrome as well. Such observation may offer a clue to a better understanding of the nature of a neurological substrate that causes RBD in this particular medical condition. The spontaneous reversibility of the symptoms in younger patients, as opposed to pattern of RBD in elderly patients, suggests that occurrence of the RBD in this group may be a result of a functional alteration of the neural pathways that control motor activity in REM sleep. Such a notion correlates with the fact that researchers have, so far, been unable to locate a site of dysfunction that could explain symptoms of the Tourette's syndrome. Recent research utilising magnetic resonance imaging showed decreased neural activation at the level of the basal ganglia. ${ }^{19}$ Symptoms of Tourette's syndrome are now generally thought to be a consequence in the cortico-striato-thalamocortical circuit. ${ }^{20}$ It has been hypothesised that Tourette's syndrome could be understood as a developmental and maturational disorder resulting in the above-mentioned dysregulation, drawing parallel to evidence that RBD may also be a maturational disorder as shown in some juvenile animal models. ${ }^{18-22}$

One of the potential shortcomings of this case report is that it was done before the concept of the paediatric autoimmune neuropsychiatric disorders associated with streptococcal infection (PANDAS) was introduced and, therefore, we do not have sufficient evidence to rule out that our patient suffered from PANDAS. The patient's clinical history was not consistent with presence of PANDAS, but no swabs were done or antibodies measured.

The differential diagnosis of frontal lobe seizures ${ }^{23}$ was carefully considered during the lengthy and extensive diagnostic process, and dismissed based on the clinical picture and EEG/PSG findings. Rapid eye movement sleep is a sleep stage generally considered as 'seizure-protective', and the pattern of the episodes (sporadic, second half of the night, associated dream mentation, 'fending' limb movements without significant tonic component) and presence of the full-spectre RBD symptoms in this patient all speak in support of the RBD diagnosis. The quality of the movements and other clinical features mentioned above excluded the nocturnal rhythmic movement disorder.

In cases such as this, one should expect complex sleep pathology, including presence of tics (reportedly present in 20\% of patients with Tourette's syndrome ${ }^{5}$ ) and (periodic) leg movements in sleep, as well as REM or non-REM sleep parasomnia, which were all present in our patient. The combination of REM and non-REM parasomnia has been previously documented, ${ }^{24}$ warranting careful clinical and polysomnographic examination before one draws definite conclusions..$^{23-25}$ Patients who also present with behavioural and psychological problems should undergo thorough and systematic psychological assessment.

\section{REFERENCES}

1. Woldrich DL, Benjamin E, Lachar D. Tourette's syndrome and psychopathology in a child psychiatry setting. J Am Acad Child Adolesc Psychiatry 1997; 36(11):1618-1624.

2. Hashimoto T, Endo S, Fukuda K, et al. Increased body movements during sleep in Gilles de la Tourette's syndrome. Brain Dev 1981; 3(1):31-35

3. Barabas G, Matthews WS, Ferrari M. Disorders of arousal in Gilles de la Tourette's syndrome. Neurology (Cleveland) 1984; 34:815817.

4. Singer HS, Walkup JT. Tourette's syndrome and other tic disorders: diagnosis, pathophysiology, and treatment. Medicine 1991; 70(1):15-18.

5. Drake ME Jr, Hietter SA, Bogner JE, Andrews JM. Cassette EEG sleep recordings in Gilles de la Tourette's syndrome. Clin Electroencephalogr 1992; 23(3):142-146.

6. Silvestri R, Raffaele M, De Domenico P, et al. Sleep features in Tourette's syndrome, neuroacanthocytosis and Huntington's chorea. Neurophysiol Clin 1995; 25 (2):66-77.

7. Rothenberger A, Kostanecka T, Kinkelbur J, et al. Sleep and Tourette's syndrome. Adv Neurol 2001; 85:245-259.

8. Voderholzer U, Muller N, Haag C, Riemann D, Straube A. Periodic limb movements during sleep are a frequent finding in patients with Gilles de la Tourette's syndrome. J Neurol 1997; 244:521526.

9. Cohrs S, Rasch T, Altmeyer S, et al. Decreased sleep quality and increased sleep related movements in patients with Tourette's syndrome. J Neurol Neurosurg Psychiatry 2001; 70(2):192-197.

10. Fish DR, Sawyers D, Allen PJ, et al. The effect of sleep on the dyskinetic movements of Parkinson's disease, Gilles de la Tourette's syndrome, Huntington's disease, and torsion dystonia. Arch Neurol 1991; 48(2):210-214.

11. Glaze DG, Frost JD Jr, Jankovic J. Sleep in Gilles de la Tourette's syndrome: disorder of arousal. Neurology (Cleveland) 1983; 133:586-592.

12. Kohyama J, Matsukura F, Kimura K, Tachibana N. Rhythmic movement disorder: polysomnographic study and summary of reported cases. Brain Dev 2002;24(1):33-38.

13. Schenck CH, Bundlie SR, Smith SA, et al. REM behavior disorder in a 10-year-old girl and aperiodic REM and NREM sleep movements in an 8-year-old brother. Sleep Res 1986; 15:162. 
14. Herman JH, Blaw ME, Steinberg JB. REM behavior disorder in a two-year-old male with evidence of brainstem pathology. Sleep Res 1989; 18:242.

15. Trajanovic NN, Shapiro CM, Sandor P. REM sleep behaviour disorder in patients with Tourette's syndrome. Sleep Res 1997;26:524.

16. Sheldon SH, Jacobsen J. REM-sleep motor disorder in children. J Child Neurol 1998;13(6):257-260.

17. Harcherik D, Leckman J, Detlor J, Cohen D. A new instrument for clinical studies of Tourette's syndrome. J Am Acad Child Psychiatry 1984 23,2:153-160.

18. Mahowald MW, Schenck CH. REM Sleep Parasomnias. In: Kryger, Roth, Dement, (Eds). Principles and Practice of Sleep Medicine. W.B. Saunders Company 2000; 64:724-741.

19. Jankovic, J. Medical Progress: Tourette's syndrome. N Engl J Med 2001; 345(16):1184-1192.
20. Gerard E, Peterson BS. Developmental processes and brain imaging studies in Tourette's syndrome. J Psychosom Res 2003; 55:13-22.

21. Singer HS. Neurobiology of Tourette's syndrome. Neurol Clin 1997; 15(2):357-379.

22. Singer HS. Current issues in Tourette's syndrome. Mov Disord 2000; 15:1051-1063.

23. Zucconi M, Oldani A, Ferini-Strambi L, Bizzozero D, Smirne S. Nocturnal paroxysmal arousals with motor behaviors during sleep: frontal lobe epilepsy or parasomnia? J Clin Neurophysiol 1997; 14(6):513-522.

24. Schenck CH, Boyd JL, Mahowald MW. A parasomnia overlap disorder involving sleepwalking, sleep terrors, and REM sleep behavior disorder in 33 polysomnographically confirmed cases. Sleep 1997;20(11):972-981.

25. Mahowald MW, Rosen GM. Parasomnias in children. Pediatrician 1990;17(1):21-31 